\title{
REMOTE SENSING OF STABLE BOUNDARY LAYER OF ATMOSPHERE
}

\author{
V.A. Banakh ${ }^{1^{*}}$, I.N. Smalikho, ${ }^{1}$, A.V. Falits ${ }^{1}$ \\ ${ }^{1}$ V.E. Zuev Institute of Atmospheric Optics SB RAS 1 Akademik Zuev Square, Tomsk, 634050, Russia \\ *Email:banakh@iao.ru
}

\begin{abstract}
The stability of the atmospheric boundary layer (ABL) has been studied experimentally with a Stream Line coherent Doppler wind lidar and an MTP-5 temperature profilometer. Twodimensional height-time distributions of the parameters characterizing the $\mathrm{ABL}$ temperature regime and wind turbulence, namely, the Richardson number, the dissipation rate of the kinetic energy of turbulence, the variance of fluctuations of the radial velocity, and the outer scale of turbulence, have been obtained. The amplitudes and period of oscillations of wind velocity components during atmospheric waves arising in the stable ABL have been determined.
\end{abstract}

\section{INTRODUCTION}

The understanding of turbulence processes in stably stratified media remains an unsolved problem in many fields of geophysics. Just this problem is a challenge to fundamental results of the Kolmogorov-Obukhov-Monin theory of isotropic turbulence, which has found wide use not only in geophysics, but also in other research fields $[1,2]$. Stable stratification usually arises when the underlying surface is cooler than the upper-lying air layers. In summer time in the atmospheric boundary layer, this occurs at night over land and in daytime over large cold water bodies. The stable stratification in the boundary layer is characterized by formation of low-level jet streams (LLJS) and atmospheric waves.

To study turbulence and temperature-wind regimes in the stable atmospheric boundary layer, we have carried out an experiment in 2018 at the west coast of Lake Baikal near the village of Listvyanka with the use of the Stream Line coherent Doppler wind lidar and the MTP-5 temperature profilometer. The experiment was conducted on August 6 till 23. Cold water of Lake Baikal provides a stability of ABL during the experiment. The results of this experiment are reported in the paper.

\section{METHODOLOGY}

The Stream Line wind lidar was installed a few meters far from the building of the Big Solar Vacuum Telescope (BSVT) at the territory of the Baikal Astrophysical Observatory of the Institute of Solar-Terrestrial Physics (ISTP) SB RAS at a distance of $340 \mathrm{~m}$ from Baikal at a height of $180 \mathrm{~m}$. The site coordinates are $52^{\circ} 50^{\prime} 47^{\prime \prime} \mathrm{N}$ and $104^{\circ} 53^{\prime} 31^{\prime \prime E}$ ). Wind measurements with the use of conical scanning by a probing beam around the vertical axis were carried out continuously during the experiment. The elevation angle of the lidar ray was $\varphi=60^{\circ}$, and the time for one complete scan (the azimuth angle $\theta$ varying from $0^{\circ}$ to $360^{\circ}$ ) was $T_{\text {scan }}=36 \mathrm{~s}$. In the most cases, the probing beam was focused to a distance of $300 \mathrm{~m}$ during the measurements. The heights from 280 to $1180 \mathrm{~m}$ above the Lake Baikal level were covered. Rough data of measurements by the wind lidar are arrays of estimates of the signal-to-noise ratio $\operatorname{SNR}\left(R_{k}, \theta_{m} ; n\right)$ and the radial velocity (projection of the wind velocity vector to the optical axis of the probing beam) $V_{L}\left(R_{k}, \theta_{m} ; n\right)$, where $R_{k}=R_{0}+k \Delta$. is the distance between the lidar and the center of the sensed volume, $k=0,1,2, \ldots, K-1, \Delta R=30 \mathrm{~m}$ is the range step, $\theta_{m}=m \Delta \theta$ is the azimuth angle, $\Delta \theta=2^{\circ}$ is the azimuth angle resolution, $m=0,1,2, \ldots M-1$, $M=180$ is the number of rays for one conical scan, and $n=1,2,3, \ldots$ is the scan number. The arrays of estimates of the radial velocities $V_{L}\left(R_{k}, \theta_{m} ; n\right)$ allow retrieval of vertical profiles of the wind velocity vector $\mathbf{V}=\left\{V_{z}, V_{x}, V_{y}\right\}$, where $V_{z}$ is the vertical component and $V_{x}, V_{y}$ are the horizontal components of the wind. Thus space-time distributions of the wind (distributions of $\mathbf{V}$ in height $h_{k}=R_{k} \sin \varphi=h_{0}+k \Delta h$ and in time $\left.t_{n}=t_{0}+(n-1) \Delta t\right)$ with the rather high vertical $\Delta h=\Delta R \sin \varphi=26 \mathrm{~m}$ and time 
$\Delta t \approx T_{\text {scan }}=36 \mathrm{~s}$ resolution are obtained. To determine the wind velocity vector, the filtered sine-wave fitting (FSWF) was used [3,4].

As a result, we have visualized the spatiotemporal distributions $U\left(h_{k}, t_{n}\right) \quad, \quad \theta_{V}\left(h_{k}, t_{n}\right) \quad$ and $V_{z}\left(h_{k}, t_{n}\right)$ for $K=30$ height levels from $h_{0}=91 \mathrm{~m}$ to $h_{K-1}=844 \mathrm{~m}$. The obtained estimates of the wind velocity vector are averaged in the scan time $T_{\text {scan }}=36 \mathrm{~s}$ and in the space along the circle of the scan cone base having the length $L_{k}=(2 \pi / \operatorname{tg} \varphi) h_{k}$, which is equal to $330 \mathrm{~m}$ at the height $h_{0}=91 \mathrm{~m}$ and $3061 \mathrm{~m}$ at the height $h_{K-1}=844 \mathrm{~m}$.

The parameters of wind turbulence (dissipation rate of turbulent energy $\varepsilon$, variance of the radial wind velocity $\sigma_{r}^{2}$, and integral scale of turbulence $L_{V}$ ) were estimated from the array of estimates of the radial velocity $V_{L}\left(R_{k}, \theta_{m} ; n\right)$ by the method of azimuth structure function (ASF) $[5,6]$. Acceptable estimates of the wind turbulent parameters by the ASF method can be obtained if the percentage of bad estimates in the array of $V_{L}\left(R_{k}, \theta_{m} ; n\right)$ is nearly zero. This restricts the height of retrieval of the wind turbulence parameters in comparison with the height of retrieval of the wind velocity vector.

To retrieve vertical profiles of wind turbulence parameters, we used the arrays of estimates of radial velocities $V_{L}\left(R_{k}, \theta_{m} ; n\right)$ obtained for 15 scans, so that the averaging time was approximately 9 minutes. As a result, we have obtained two-dimensional height-time distributions $\varepsilon\left(h_{k}, t_{n}\right), \sigma_{r}^{2}\left(h_{k}, t_{n}\right), \quad$ and $L_{V}\left(h_{k}, t_{n}\right)$ for $K=17$ height levels from $h_{0}=91 \mathrm{~m}$ to $h_{K-1}=506 \mathrm{~m}$.

The MTP-5 temperature profilometer was installed at the top of a hill near the foot of BSVT.
The profilometer allows us to obtain the vertical temperature profiles every three minutes with a resolution of $25 \mathrm{~m}$ for heights from 0 to $100 \mathrm{~m}$ and $50 \mathrm{~m}$ for heights from $100 \mathrm{~m}$ to $1000 \mathrm{~m}$. The profilometer data were used to obtain the spatiotemporal distributions of the mean temperature of air $T(h, t)$, its derivative $\partial T(h, t) / \partial h$, and potential temperature $T_{p}(h, t)=T(h, t)+\gamma_{a} h+C_{1} \quad, \quad$ where $\gamma_{a}=0.01 \mathrm{deg} / \mathrm{m}$ is the dry-adiabatic gradient $\left(\partial T_{p}(h, t) / \partial h=\partial T(h, t) / \partial h+\gamma_{a}\right) \quad$. For temperature averaging, we used the data measured for $24 \mathrm{~min}$. The averaged data were used to calculate the parameter

$$
N^{2}=\frac{g}{T_{p}} \frac{\partial T_{p}}{\partial h}
$$

which characterizes the thermal stratification of the atmosphere, where $g$ is the free-fall acceleration. The stratification is unstable at $N^{2}<0$, neutral at $N^{2}=0$, and stable at $N^{2}>0$. In the case of stable stratification, $N$ is the Brunt-Vaisala frequency.

The Richardson number Ri was calculated as

$$
\mathrm{Ri}=N^{2}\left(\frac{\partial U}{\partial h}\right)^{-2}
$$

where lidar data for the horizontal components of the wind vector $V_{x}$ and $V_{y}$ averaged over 31 scans $(20 \mathrm{~min})$ were used to obtain the average wind velocity $U$ and its derivative $\partial U / \partial h$.

\section{RESULTS}

It follows from the obtained temperature data that the conditions for stable stratification occurred in the boundary layer during the entire period of measurements in August 6 to 23. The vertical temperature gradient always exceeded the dryadiabatic one. Figure 1 shows the spatiotemporal distributions of the wind velocity and the Richardson number for August 21 to 23 as obtained from measurements with the wind lidar and the temperature profilometer. 


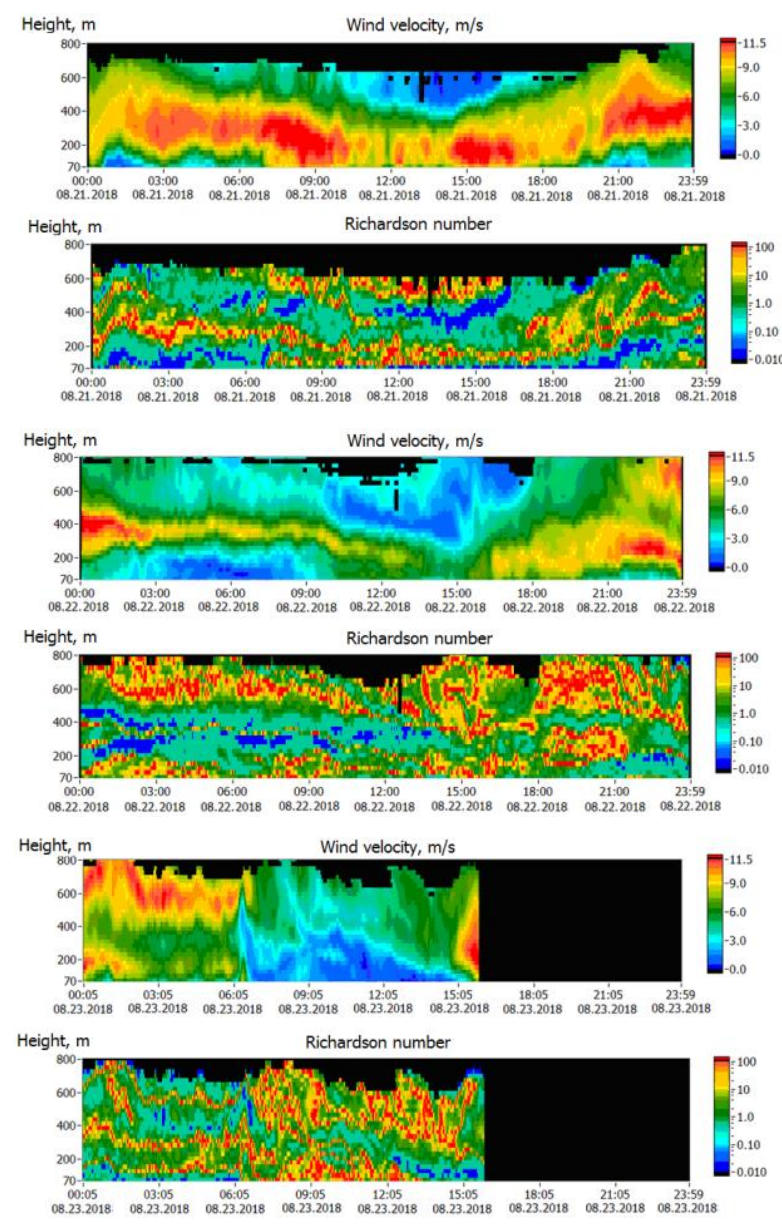

Figure 1 - Spatiotemporal distributions of the wind velocity and the Richardson number for August 21-23 of 2018.

It can be seen from the figure that jet streams were observed in the boundary layer of the Baikal coastal zone during this period. Two jet streams were observed at the night of August 22 to 23 and in the daytime of August 23. A characteristic feature of the data shown in Figure 1 is that beyond streams the Richardson number takes large positive values far exceeding the critical value $\mathrm{Ri}_{\mathrm{cr}}=0.25$, which is indicative of the high stability of the boundary layer. At the heights of location of jet streams, the Richardson number takes much smaller values. It still keeps positive, but its value becomes smaller than $\mathrm{Ri}_{\text {cr. }}$. That is, in the region of jet streams, the stability of thermal stratification of the boundary layer decreases, and channels arise, in which the conditions are close to neural stratification.
Atmospheric waves may appear in the stable boundary layer simultaneously with jet streams. Among all the data measured by the Stream Line lidar in August 6 to 23 of 2018, we revealed 14 cases of atmospheric wavy formation. From the obtained distributions $U\left(h_{k}, t_{n}\right), \theta_{V}\left(h_{k}, t_{n}\right)$, and $V_{z}\left(h_{k}, t_{n}\right)$, we first determined visually the heights and time of appearance of periodic variations in the wind velocity components. Then we determined the parameters of atmospheric waves by the method described in [7]. In all the cases of observation of atmospheric waves, the amplitude of the wave component of the horizontal wind velocity $A_{v}$ was no smaller than $0.6 \mathrm{~m} / \mathrm{s}$. The amplitude of oscillations of the vertical velocity was no smaller than $0.2 \mathrm{~m} / \mathrm{s}$. The maximal amplitudes of wind oscillations were seen in the wave observed from 02:30 to 03:30 LT on August 23 of 2018. They amounted to $A_{v} \approx 3 \mathrm{~m} / \mathrm{s}$ for the horizontal wind velocity component and $A_{v} \approx 1 \mathrm{~m} / \mathrm{s}$ for the vertical one.

The lifetime of atmospheric waves observed in the region under study in August 6 to 23 of 2018 varied from $40 \mathrm{~min}$ to 5 hours. The oscillation period ranged from 5 to $20 \mathrm{~min}$. This oscillation period far exceeds the Brunt-Vaisala frequency (1) calculated from the data of temperature profilometer. This means that the mountain relief of the Baikal coastal zone is critical for formation of atmospheric waves observed in our experiment.

Figure 2 exemplifies the height-time distribution of wind turbulence parameters retrieved from lidar measurements of the radial velocity on August 21 of 2018. At that day, jet streams were observed from 00:00 to 08:30 LT and from 21:00 to 24:00 LT at heights of $200-400 \mathrm{~m}$. It can be seen from Figs. $2(a, b)$ that turbulence is quite weak at the heights of location of jet streams. To the contrary, turbulence is very strong beyond jet streams, at heights of $100-200 \mathrm{~m}$. At these heights, the dissipation rate $\varepsilon$ achieves $0.09 \mathrm{~m}^{2} / \mathrm{s}^{3}$, while the variance $\sigma_{r}^{2}$ is $2.5(\mathrm{~m} / \mathrm{s})^{2}$. An atmospheric wave formed approximately at 7 a.m. for the short 40 min time interval. An increase in the turbulence intensity from 07:00 to 09:00 LT at heights exceeding $350 \mathrm{~m}$ is likely connected with the destruction of this atmospheric wave. 


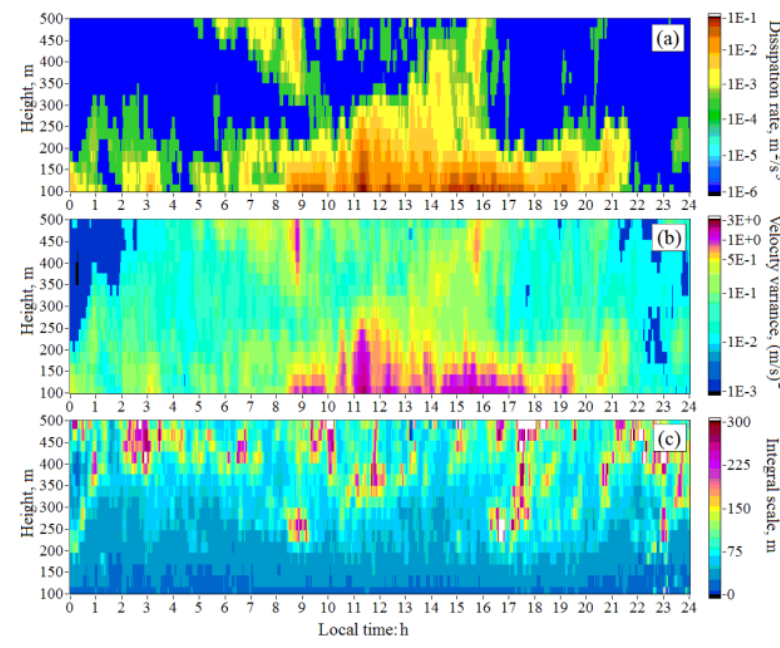

Figure 2 - Height-time distribution of the dissipation rate of turbulence energy (a), variance of the radial wind velocity (b) and the integral scale of turbulence (c) obtained from measurements by the Stream Line lidar on August 21 of 2018.

The analogous distributions $\varepsilon\left(h_{k}, t_{n}\right), \sigma_{r}^{2}\left(h_{k}, t_{n}\right)$, and $L_{V}\left(h_{k}, t_{n}\right)$ obtained on August 23 of 2018 demonstrate that during the atmospheric waves observed at that day from $02: 30$ to $03: 30$, from 05:30 to $06: 30$, and from 08:30 to 09:30 LT, the intensity of wind turbulence was different at different heights in ABL. The atmospheric wave from 02:30 to 03:30 formed in the presence of two jet streams (see Fig. 1), the angle between the directions of wind flows in which was approximately $90^{\circ}$. In this wave, the amplitudes of oscillation of wind velocity components were maximal for the entire period of measurements.
The oscillation period was identical at all the heights of lidar measurements, in particular, at the heights of location of the both jet streams. The dissipation rate of the turbulence energy in the lower jet stream appeared to be an order of magnitude smaller than that at the heights of location of the upper jet stream. As the wave disappeared, the turbulence became moderate at all the heights. Thus, during atmospheric waves, the wind turbulence may be both moderate and even weak and strong.

\section{ACKNOWLEDGEMENTS}

This study was performed under project №AAAA-A17-117021310149-4 and supported, in part, by the Russian Foundation for Basic Research, Project No. 18-05-00220.

\section{REFERENCES}

[1] G.S. Golitsyn, Statistics and Dynamics of Natural Processes and Phenomena: Methods, Instruments, and Results (Krasand, Moscow, 2013), 400 pp.

[2] G.S. Golitsyn, Gidrol. Meteorol. 3, 5-15 (2018)

[3] V.A. Banakh, et al. Atmospheric and Oceanic Optics 23(5): 381-388 (2010)

[4] V.A. Banakh, et al. Atmospheric and Oceanic Optics 28(2): 185-191 (2015)

[5] I.N. Smalikho, V.A. Banakh, Atmospheric Measurement Techniques. 10(11): 4191-4208 (2017)

[6] V.A. Banakh, I.N. Smalikho, A.V. Falits, Optics Express 25(19): 22679-22692 (2017)

[7] V.A. Banakh, I.N. Smalikho, Atmospheric Measurement Techniques 9(10): 5239-5248 (2016) 